\title{
A New Proof of Parisi's Conjecture for the Finite Random Assignment Problem
}

\author{
Chandra Nair \\ Dept. of Electrical Engineering \\ Stanford University \\ Stanford, CA 94305-9510 \\ e-mail: mchandra@stanford.edu
}

\author{
Balaji Prabhakar \\ Depts. of Elec. Engg. \& Comp. Science \\ Stanford University \\ Stanford, CA 94305-9510 \\ e-mail: balaji@ee.stanford.edu
}

\author{
Mayank Sharma \\ Dept. of Electrical Engineering \\ Stanford University \\ Stanford, CA 94305-9510 \\ e-mail: msharma@stanford.edu
}

\begin{abstract}
Consider the problem of minimizing cost when assigning $n$ jobs to $n$ machines. An assignment is a one-to-one mapping of jobs onto the machines. Assume that the cost of executing job $i$ on machine $j$ is $c_{i j}, i, j=1, \ldots, n$. When the $c_{i j}$ are i.i.d. exponentials of mean 1, Parisi conjectured that the average cost of the minimum assignment equals $\sum_{i=1}^{n} \frac{1}{i^{2}}$. Recently, the authors, and independently, Linusson and Wästlund, have proved this conjecture. In the above work the authors also made a refined conjecture that, if established, would yield another proof of the Parisi's conjecture. This paper establishes the refined conjecture, thus providing a new proof of Parisi's conjecture.
\end{abstract}

\section{INTRODUCTION}

Consider a system with $n$ jobs and $n$ machines where the cost of executing job $i$ on machine $j$ is $c_{i j}$. The assignment problem concerns the determination of a 1-to-1 assignment of jobs onto machines that minimizes the cost of executing all the jobs. The cost of the minimizing assignment is given by $A_{n}=$ $\min _{\pi} \sum_{i=1}^{n} c_{i, \pi(i)}$. In the random assignment problem the $c_{i j}$ are i.i.d. random variables drawn from some distribution, and the quantity of interest is the expected minimum cost, $\mathbb{E}\left(A_{n}\right)$. For $c_{i j} \sim$ i.i.d. $\exp (1)$ variables, Parisi [9] conjectured that:

$$
\mathbb{E}\left(A_{n}\right)=\sum_{i=1}^{n} \frac{1}{i^{2}} .
$$

Let $C=\left[c_{i j}\right]$ be an $n \times n$ cost matrix with i.i.d. $\exp (1)$ entries. Delete the top row of $C$ to obtain the rectangular matrix $L$ of dimensions $(n-1) \times n$. For each $i=1, \ldots, n$, let $S_{i}$ be the cost of the minimum-cost permutation in the sub-matrix obtained by deleting the $i^{t h}$ column of $L$. These quantities are illustrated below.

\begin{tabular}{|l|l|l|}
\hline 3 & 6 & 11 \\
\hline 9 & 2 & 20 \\
\hline \multicolumn{3}{|c|}{$\mathrm{L}$}
\end{tabular}

\begin{tabular}{|c|c|}
\hline 6 & $\mathbf{1 1}$ \\
\hline $\mathbf{2}$ & 20 \\
\hline \multicolumn{2}{|c|}{$S_{1}=13$}
\end{tabular}

\begin{tabular}{|l|c|}
\hline 3 & $\mathbf{1 1}$ \\
\hline $\mathbf{9}$ & 20 \\
\hline \multicolumn{2}{|c|}{$S_{2}=20$}
\end{tabular}

\begin{tabular}{|c|c|}
\hline $\mathbf{3}$ & 6 \\
\hline 9 & $\mathbf{2}$ \\
\hline \multicolumn{2}{|c|}{$S_{3}=5$} \\
\hline
\end{tabular}

Let $\sigma$ be the random permutation of $\{1, \ldots, n\}$ such that $S_{\sigma(1)} \leq \ldots \leq S_{\sigma(n)}$. Define $T_{i}=S_{\sigma(i)}$. We shall refer to the sequence $\left\{T_{i}, i=1, \ldots, n\right\}$ as the $T$-matchings of $L$. In the above example, $T_{1}=5, T_{2}=13$ and $T_{3}=20$.

In [8] we prove the following

Theorem 1 For $j=1, \ldots, n-1, \quad T_{j+1}-T_{j} \sim \exp (j(n-j))$ and these increments are independent of each other.

Theorem $2 \mathbb{E}\left(A_{n}\right)=\sum_{i=1}^{n} \frac{1}{i^{2}}$.

In [8], we use Theorem 1 to establish Theorem 2.

\section{MAIN RESUlT}

Let $L$ be an $(n-1) \times n$ matrix of i.i.d. $\exp (1)$ entries and let $\left\{T_{i}\right\}_{1}^{n}$ denote its $T$-matchings, as defined in the previous section. Let $\Upsilon$ denote the set of all placements of the row-wise minimum entries of $L$; for example, all the row-wise minima in the same column, all in distinct columns, etc. Now consider any fixed placement of the row minima $\xi \in \Upsilon$. We prove the following conjecture made in [8]

Theorem 3 Conditioned on a particular placement $\xi$,

$$
T_{j+1}-T_{j} \sim \exp (j(n-j)) \text { for } j=1, \ldots, n-1 .
$$

Furthermore, these increments are independent of each other.

The proof of Theorem 3 uses the memoryless property of the exponential distribution and some combinatorial observations to reduce the computations to that of Theorem 2 .

Clearly, if we average over all $\xi \in \Upsilon$ then we recover Theorem 1. Hence Theorem 3 is a refinement of Theorem 1. It turns out that Theorem 3 is simple to prove in the case when $\xi$ is the placement corresponding to all row-wise minima being in distinct columns.

\section{REFERENCES}

[1] D. J. Aldous. Asymptotics in the random assignment problem, Probab. Th. Rel. Fields, 93 (1992) 507-534.

[2] D. J. Aldous. The $\zeta(2)$ Limit in the Random Assignment Problem, Random Structures and Algorithms 18 (2001) 381-418.

[3] D. Coppersmith and G. Sorkin. Constructive bounds and exact expectations for the random assignment problem, Random Structures and Algorithms, 15 (1999) 113-144.

[4] S. Linusson and J. Wästlund. A Generalization of the Random Assignment Problem, arXiv: math.CO/0006146.

[5] S. Linusson and J. Wästlund. A Proof of Parisi's Conjecture on the Random Assignment Problem, accepted for publication in Probab. Th. Rel. Fields. http://www.mai.liu.se/ svlin/rap.html

[6] M. Mézard and G. Parisi. On the solution of the random link matching problem, J. Physique, 48 (1987) 1451-1459.

[7] M. Mézard, G. Parisi and M. A. Virasoro. Spin Glass Theory and Beyond, World Scientific, Singapore, 1987.

[8] C.Nair, B. Prabhakar and M. Sharma. A Proof of the Conjecture due to Parisi for the Finite Random Assignment Problem, submitted. http://www.stanford.edu/ mchandra

Conference version: Proofs of the Parisi and CoppersmithSorkin Conjectures for the Finite Random Assignment Problem, Proceedings of IEEE FOCS, 2003.

[9] G. Parisi. A conjecture on random bipartite matching, Technical Report cond-mat/9801176, XXX LANL Archive, 1998

[10] J. Michael Steele. Probability Theory and Combinatorial Optimization. Number 69 in CBMS-NSF Regional Conference Series in Applied Mathematics. SIAM, Philadelphia PA, 1997. 